\title{
Haptically Annotated Movies: Reaching Out and Touching the Silver Screen
}

\author{
Derek Gaw, Daniel Morris, and Kenneth Salisbury \\ Computer Science Department, Stanford University \\ \{dgaw,dmorris\}@cs.stanford.edu, jks@robotics.stanford.edu
}

\begin{abstract}
The film industry consistently strives to make the movie-going experience more immersive and more captivating, through larger screens, higher-quality images, and increasingly sophisticated speaker systems. Currently, however, presentation of movies is limited to the visual and auditory senses. Haptics provides significant potential for augmenting the theater experience beyond those sensory modalities. In this paper and the accompanying demo, we present a system for recording and annotating haptic information that is time-referenced to a movie, then replaying the recorded haptic information to a user. We discuss several user interface issues that we addressed and several scenarios that are augmented by this system.
\end{abstract}

\section{Introduction and Related Work}

In order to increase cinematic immersiveness, movie theaters are turning to higher-resolution formats and larger screens and are using larger numbers of speakers with greater sound fidelity. Similar trends are occurring in home electronics, bringing higher fidelity to the visual and auditory aspects of home television. However, other senses - specifically touch - are still largely ignored in commercial video.

Force-feedback has begun to make its way into other forms of display-based entertainment. Amusement parks employ a large-scale form of haptic feedback in the form of hydraulic platforms. Movements of an entire room are time-linked to a video presentation to create the illusion of movement with the camera, often to simulate vehicle motion (e.g. the "Back to the Future" ride at Universal Studios).

Additionally, one- and two-degree-of-freedom haptics have seen significant success in the video game industry, with force-feedback devices available at consumer prices and supported by numerous games. Similarly, O'Modhrain and Oakley [1] enhanced cartoon animations with simple haptic interactivity.

But to date, no previous work has connected finegrained haptic feedback to conventional video media. We have approached this problem by developing an authoring environment for embedding haptic information in a video stream, providing an author with several tools for editing haptic force and position trajectories. A user then grasps a haptic device while viewing the video and receives haptic feedback at scenes selected by the author.

Several groups have presented pre-recorded visuohaptic paths to subjects to teach specific motor skills [2-4]. Adams et al [5] assessed the value of haptic training in conjunction with instructional video.

\section{Design Challenges}

The goal of haptically annotating movies is to allow the viewer (now also a user of the haptic device) to feel what happens on screen; however, it is not initially clear how such interaction should take place. Should a viewer be able to pause the movie and probe the environment with the device? What should happen during a dramatic dialogue, when haptic feedback is not relevant to the primary content of the movie?

We accept that haptic interaction is not applicable to all movie styles. There also exists an inherent incompatibility between haptics and traditional movies: the former is typically an interactive medium while the latter is not. Both real-world and virtual haptics involve feedback from the environment based on constant user input. However, the data available about the environment displayed in a movie is insufficient to allow this type of probe/response approach to haptic interaction. Thus the viewer must be guided by the movie rather than initiating his own movements.

In spite of this distinction, there are numerous situations that we expect would be complemented by haptic data. Consider, for example, a sword duel during which a viewer could experience the forces felt by one of the actors, or a chase scene during which the viewer could feel the vehicle's controls and feedback.

\section{Implementation}

Our environment makes use of two graphical interfaces: one for recording haptic information and one for playing it back. The primary stream of information is a series of positions and orientations which will be played back with a PD controller. This information is augmented with explicit force information in situations where an author determines that positional data is inadequate. 


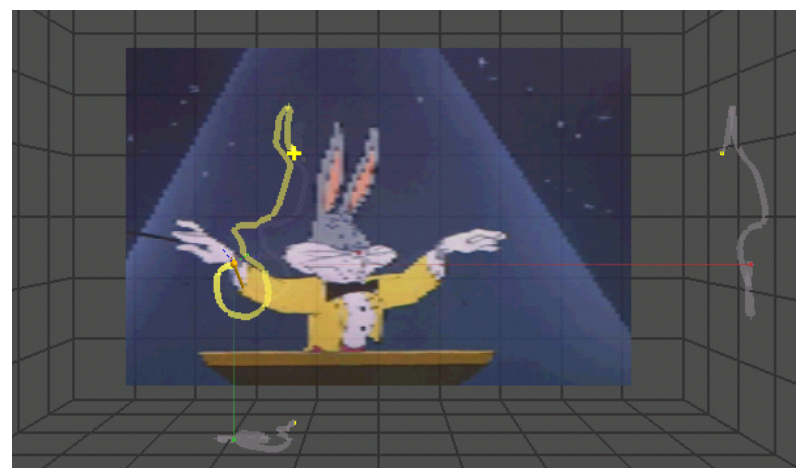

Figure 1. An author edits a haptic motion path to match the movement of the animated character's hand. The path is projected onto the right and bottom walls for precise positioning.

For the purpose of recording, we present an axisaligned box that approximates the haptic device's workspace, in which we display a planar representation of the video being annotated and a graphical representation of the current haptic trajectory (Figs 1 and 2). Semitransparent video allows the user to view the video and the haptic trajectory simultaneously.

Reference lines from the virtual probe to each of the axis planes allow precise positioning of the device. When the author initiates recording, video playback begins from a selected frame, and the position and orientation of the haptic device are sampled at $1 \mathrm{kHz}$. Recording a haptic path at the actual speed of a video may be difficult for an author, particularly when the desired path moves quickly and subtle movements are required. We thus allow the trajectory to be defined while the video is presented at a reduced speed.

It is also difficult to approximate sharp impulses with position data alone. To address this issue, an impulse - defined by duration, magnitude, direction, and shape - can be added to the recorded path. Each impulse applies a force vector to the device that will be added to the constraining (PD-control) force during playback.

A challenge with the recording environment is visualizing the haptic path without introducing excess noise and information overflow. We found that simply rendering the path itself as a series of solid line segments can be confusing. For this reason, the path is "onion-skinned" to only show a time-range immediately trailing the current time. Other portions of the path are dimmed to reduce irrelevant information. The device path is also projected onto each of the planes of the world to facilitate positioning. Force impulses are represented as transparent icons attached to the path.

When a user initiates playback, the device is initially constrained to the path's starting position. When the

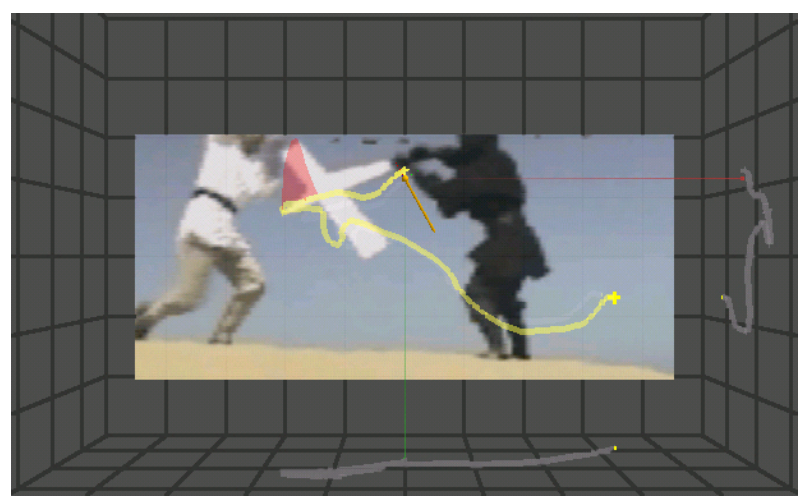

Figure 2. An author edits a haptic motion path to match the hand movement of the character on the right. The red triangle indicates a force pulse that was added to explicitly model a collision.

device reaches this position, haptic and video playback begin simultaneously, and a PD controller guides the user along the recorded path.

\section{Future Work}

Future work will focus on empirical evaluation of the subjective experience provided by our system and the clarity of the interface available to authors. We also plan to examine the applicability of our system to nonentertainment media, particularly the coupling of surgical training videos with trajectories recorded by experienced surgeons.

The work presented here depends on an author to manually annotate the video with haptic information; we hope to explore the use of computer vision techniques to automatically generate motion paths. Similarly, we hope to make use of other data that may be available during filming - e.g. motion capture information or polygonal environment models used for special effects - to further enhance haptic playback.

\section{References}

[1] S. O’Modhrain and I. Oakley, "Adding Interactivity: Active Touch in Broadcast Media”, $12^{\text {th }}$ IEEE Haptics Symposium, Mar 2004.

[2] D. Feygin, M. Keehner, and F. Tendick, "Haptic Guidance: Experimental Evaluation of Haptic Training Method for a Perceptual Motor Skill”, $10^{\text {th }}$ IEEE Haptics Symposium, Mar 2002.

[3] R.B. Gillespie, M. O’Modhrain, P. Tang, D. Zaretzky, and C. Pham, “The Virtual Teacher”, ASME International Mech Eng Congress and Exposition, Nov 1998.

[4] R.L. Williams, M. Srivastava, and J. Howell, "Implementation and Evaluation of a Haptic Playback System”, Haptics-e, Vol 3.3, May 2004.

[5] R.J. Adams, D. Klowden, B. Hannaford, "Virtual Training for a Manual Assembly Task”, Haptics-e, Vol 2.2, Oct 2001. 